\title{
Rolling Force Prediction in Heavy Plate Rolling Based on Uniform Differential Neural Network
}

\author{
Fei Zhang, ${ }^{1,2}$ Yuntao Zhao, ${ }^{3}$ and Jian Shao ${ }^{1}$ \\ ${ }^{1}$ National Engineering Research Center for Advanced Rolling Technology, University of Science and Technology Beijing, \\ Beijing 100083, China \\ ${ }^{2}$ School of Electrical, Computer \& Telecommunications Engineering, University of Wollongong, Wollongong, NSW 2522, Australia \\ ${ }^{3}$ WISDRI (Wuhan) Automation Co. Ltd., Wuhan 430223, China
}

Correspondence should be addressed to Fei Zhang; zhfeicn@gmail.com

Received 31 March 2016; Accepted 29 May 2016

Academic Editor: Roberto Sabatini

Copyright (C) 2016 Fei Zhang et al. This is an open access article distributed under the Creative Commons Attribution License, which permits unrestricted use, distribution, and reproduction in any medium, provided the original work is properly cited.

\begin{abstract}
Accurate prediction of the rolling force is critical to assuring the quality of the final product in steel manufacturing. Exit thickness of plate for each pass is calculated from roll gap, mill spring, and predicted roll force. Ideal pass scheduling is dependent on a precise prediction of the roll force in each pass. This paper will introduce a concept that allows obtaining the material model parameters directly from the rolling process on an industrial scale by the uniform differential neural network. On the basis of the characteristics that the uniform distribution can fully characterize the solution space and enhance the diversity of the population, uniformity research on differential evolution operator is made to get improved crossover with uniform distribution. When its original function is transferred with a transfer function, the uniform differential evolution algorithms can quickly solve complex optimization problems. Neural network structure and weights threshold are optimized by uniform differential evolution algorithm, and a uniform differential neural network is formed to improve rolling force prediction accuracy in process control system.
\end{abstract}

\section{Introduction}

The steel plates are used for applications such as shipbuilding, bridge construction, civil engineering, industrial machinery, and offshore structures, which require high quality and high strength for reliability. Recently, market demands are growing increasingly strict for high-quality products in hot rolling. Particularly, the requirement for thickness precision in a rolling mill process is stricter than any other request. Good thickness precision is highly related with good rolling force prediction [1]. A typical process as shown in Figure 1 mainly consists of two furnaces, a rolling stand and a cooling system.

A plate-making process goes through the following steps. Initially, a slab is reheated to recrystallization temperature (about $1215-1230^{\circ} \mathrm{C}$ ) in the furnace, and it is rolled to a final target plate after about a dozen passes in the plate mill. Then, the microstructure of plate is controlled by the phase transformation of austenite during the cooling processes.

After the slab's extraction from the furnace, the operation sequences in the rolling section are determined by a pass calculation algorithm, which calculates the sequences of rolling operations required and predicts the characteristics of the plate after each pass. Figure 2 shows the rolling sequences in each pass which consists of three steps: the precalculation step, the real-time control step (or rolling phase), and the postcalculation step [1].

The plate rolling process is a complicated process with multiple variables, nonlinearity, and strong coupling. Because of the complexity of rolling environment, such as the changes of material constant, friction coefficient, surface roughness of roller, roll wear, oil film thickness, and lubrication condition, the set calculation results of the rolling force, rolling torque, front slide, and deformation resistance are different from the actual rolling process. The rolling force is the most important equipment parameter and technological parameter of rolling mill, for it is the important basis of plastic processing technology, equipment optimization design, and process control. The calculation accuracy of the rolling force directly affects the setting accuracy of the rolling schedule; besides, it is the key to make full use of the regulatory capacity of the thickness and 


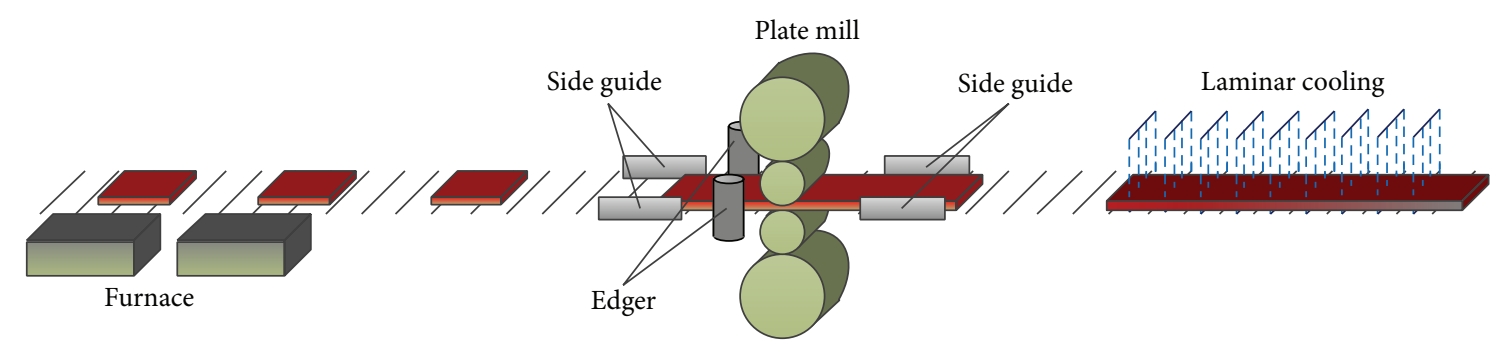

Figure 1: Plate mill scheme.

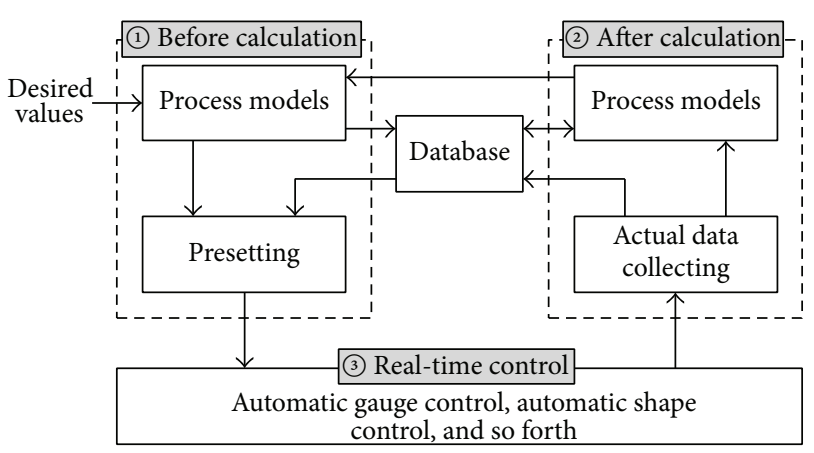

FIgURE 2: Process control system of a plate mill.

shape control system and the key to improve the hit rate of the steel head. As the conventional rolling force is calculated by the rolling force mathematical model based on experience and statistics, there are some defects in the process of using. Firstly, for the purposes of online control, the general mathematical model is simplified under certain assumptions, so it cannot provide sufficiently accurate predictive value. Secondly, because of the variation of the measurement errors and system characteristics, the parameter errors of model are also great. Therefore, in order to improve the accuracy of rolling force setting, adaptive and self-learning methods based on instant information are used to modify the model [2].

Rolling schedule plays an important role in the process of plate rolling production. And an excellent rolling schedule is the basic guarantee for the production capacity of rolling mill, for it can improve the quality of products. The medium and thick plate rolling schedule mainly includes the reduction (load) system, the speed system, the temperature system, and the roller type system. Based on the technical requirements of steel, raw material conditions, temperature conditions, and the actual situation of production equipment, rolling schedule design can make artificial calculation or computer calculation to determine the actual reduction, no-load roll gap, rolling speed, and other parameters with the use of mathematical formulas or charts; in the meanwhile, according to the adaptive correction and processing under condition of actual rolling, rolling schedule design can give full play to the equipment potential, increase production, guarantee quality, make operation easy, and make equipment safe.

To develop the correct rolling schedule, a reasonable reduction (load) distribution must be determined. Because of the characteristics of the plate rolling, the research on load distribution started very late, but whether it is the traditional optimization method or intelligent optimization method, the whole process of optimization is generally summarized as 4 steps: (1) Determine the objective function of rolling load distribution according to the actual production conditions; (2) determine the constraint conditions according to the actual production conditions; (3) choose the appropriate optimization method; (4) derive the extreme value of the objective function and obtain the process parameters when the objective function reaches its extreme value.

\section{Mathematical Model of Plate Rolling}

2.1. Rolling Force Model. The rolling force model is in the core position in the plate model system, as it is an important parameter to develop technological system, adjust the mill, improve the product quality, expand the product range, fully and reasonably tap equipment potential, and check equipment strength. At present, Sims' model is recognized as the most suitable model for hot rolling [3]. Sims' model assumes the plate deformation to be purely plastic. In consideration of the yield condition derived by Orowan and making some assumptions on the friction between the work rolls and the plate, Sims calculates the rolling force $P$ by integrating Von Karman's differential equation [4]:

$$
P=B l_{c}^{\prime} Q_{P} K K_{T},
$$

where $P$ is the rolling force, $B$ is the plate width, $l_{c}^{\prime}$ is the horizontal projection length of contact arc of the squashed roller and the rolling plate, $Q_{P}$ is the influence coefficient of friction force in the contact arc, $K$ is the metal deformation resistance, and $K_{T}$ is the effect factor of rolling force by tensile stress.

2.2. Rolling Torque Model. Rolling torque can be determined by rolling force or energy consumption:

$$
M=2 \times F \times \sqrt{R^{\prime} \times \Delta h} \times \psi_{p},
$$

where $\psi_{p}$ is the moment arm coefficient, whose value in plate rolling is 0.4 to 0.8 in general, and it can also be calculated by the following empirical formula [5]:

$$
\psi_{p}=0.5-0.028 \frac{\sqrt{R^{\prime} \times \Delta h}}{h_{0}} .
$$


The rolling power is related to the rolling torque and the roll speed:

$$
P=M \times \frac{n}{971},
$$

where $P$ is the rolling power, $M$ is the rolling torque, and $n$ is the roll speed.

2.3. Temperature Drop Model. Temperature is one of the most influential factors to the deformation resistance. With the increase of temperature, the strength indexes, including yield limit, strength limit, and hardness, of all kinds of metals and alloys will decrease. This is because, with the increase of temperature, the amplitude of the thermal agitation of the metal atom increases and the bond force between the atoms decreases; accordingly the needed energy and the deformation resistance of the metal plastic deformation decrease. Generally plate temperature model includes the temperature drop of radiation, descaling, and interstand cooling water when the rolling plate is conveyed on the roll table or in the mill housing, the temperature rise of rolling deformation, and the temperature drop of contact between the rolling plate and the roll table or roller [3]. The radiation temperature drop model takes the formula in [6], and models of the descaling temperature drop, the interstand cooling water temperature drop, rolling deformation temperature rise, and the contact temperature drop take the formula in [3].

\section{Differential Evolution Algorithm}

DE (differential evolution) algorithm proposed by Storn and Price in 1995 is a random group evolutionary search algorithm, which can guide the optimization process by the swarm intelligence produced by individual cooperation and competition. Due to the ease of use, robustness, and strong global optimization ability, DE algorithm has been successfully used in many fields [7-13]. On the basis of the traditional mathematical model, this paper studies how to improve the accuracy of rolling force prediction by using DE algorithm.

When DE algorithm is solving optimization problems, the parallel search will be completed by $N_{P}$ individuals together in the search space. The basic operation of the DE includes mutation, crossover, and selection. The initial population is randomly generated in the search space, and it is generally produced by a uniform distribution of random functions. In case a preliminary solution is available, the initial population might be generated by adding normally distributed random deviations to the nominal solution. DE generates new parameter vectors by adding the weighted difference between two population vectors to a third vector. This operation is called mutation. The mutated vector's parameters are then mixed with the parameters of another predetermined vector, the target vector, to yield the so-called trial vector. Parameter mixing is often referred to as "crossover." If the fitness of the trial vector is better than the fitness of the target vector, the trial vector replaces the target vector in the following generation. This operation is called selection $[14,15]$.
Set $N_{P} D$-dimensional real vectors as each generation population; each individual can be expressed as

$$
x_{i}^{g}, \quad\left(i=1,2, \ldots, N_{P}\right),
$$

where $i$ is the sequence of individual in population, $g=$ $1,2, \ldots, G$ is the evolutionary generation, $N_{P}$ is population size, and $N_{P}$ does not change during the evolution process [16].

\subsection{Basic Differential Evolution Algorithm}

3.1.1. Initialization. In order to establish the initial point of optimal search, the population should be initialized first. One method of generating the initial population is randomly chosen from the given boundary constraints. In the DE research, it is generally assumed that all the randomly initialized populations accord with uniform probability distribution.

Set parameter variable boundary as $x_{i j}^{(L)} \leq x_{i j} \leq x_{i j}^{(U)}$; then

$$
x_{i j}(0)=\operatorname{rand}[0,1] \cdot\left(x_{i j}^{(U)}-x_{i j}^{(L)}\right)+x_{i j}^{(L)},
$$

where $i=1,2, \ldots, N_{P}, j=1,2, \ldots, D$, and $\operatorname{rand}[0,1]$ is a random number in the range of $[0,1]$.

3.1.2. Mutation. For each target individual $x_{i}^{g}, i=1,2, \ldots$, $N_{P}$, a mutant individual is generated according to

$$
v_{i}^{g}=x_{r_{1}}^{g}+F\left(x_{r_{2}}^{g}-x_{r_{3}}^{g}\right)
$$

with random indexes $r_{1}, r_{2}, r_{3} \in\left\{1,2, \ldots, N_{P}\right\}$, which are mutually different and $F>0$. The randomly chosen integers $r_{1}, r_{2}$, and $r_{3}$ are also chosen to be different from the running index $i$, so $N_{P} \geq 4$ must be satisfied. The mutation operator $F \in[0,2]$ is a real and constant factor which controls the amplification of the differential variation $\left(x_{r_{2}, G}-x_{r_{3}, G}\right)$. So far the study shows that $F$ less than 0.4 and more than 1 is only occasionally effective, and $F=0.5$ is usually a good initial choice.

3.1.3. Crossover. In order to increase the diversity of the population, the crossover operation is introduced. Variant individual $v_{i}^{g}$ and the corresponding target individual $x_{i}^{g}$ obtained from the mutation operation generate trial vector $u_{i}^{g}$ according to

$$
u_{i}^{g}= \begin{cases}v_{i j}^{g}, & \text { if } \operatorname{rand} b(j) \leq \mathrm{CR} \text { or } j=\operatorname{mbr}(i) \\ x_{i j}^{g}, & \text { if } \operatorname{rand} b(j)>\mathrm{CR} \text { or } j \neq \operatorname{mbr}(i)\end{cases}
$$

where $u_{i}^{g}=\left(u_{i 1}^{g}, u_{i 2}^{g}, \ldots, u_{i D}^{g}\right), i=1,2, \ldots, N_{P}, j=1,2, \ldots$, $D$, rand $b(j)$ is the $j$ th evaluation of a uniform random number generator with outcome $[0,1], \operatorname{mbr}(i) \in 1,2, \ldots, D$ is a randomly chosen index which ensures that $u_{i}^{g}$ obtains at least one parameter from $v_{i}^{g}$, and CR $\in[0,1]$ is the crossover constant.

Formula (8) indicates that if CR becomes greater, the contribution of $v_{i}^{g}$ to $u_{i}^{g}$ is greater; if CR $=1$, then $u_{i}^{g}=v_{i}^{g}$, which 
is advantageous to local search and accelerates convergence rate; if CR becomes less, the contribution of $x_{i}^{g}$ to $u_{i}^{g}$ is greater; if CR $=0$, then $u_{i}^{g}=x_{i}^{g}$, which is beneficial to maintain the diversity and global search.

3.1.4. Selection. In order to ensure that the better individual is selected into the next generation, DE compares the trial vector $u_{i}^{g}$ and the target individual $x_{i}^{g}$ in the current population according to the principle of greed. If the objective function is minimized, the vector with the smaller objective function values will win a place in the next generation:

$$
x_{i}^{g+1}= \begin{cases}u_{i}^{g}, & \text { if }\left(u_{i}^{g}\right)<f\left(x_{i}^{g}\right) \\ x_{i}^{g}, & \text { otherwise. }\end{cases}
$$

3.1.5. Boundary Handling. In the problem of boundary constraints, it is necessary to make sure that the new individual is located in the feasible region of the problem, and a simple method is replaced by the random generation of the new individual with the feasible region.

$$
\begin{aligned}
& \text { That is, if } x_{i j}^{g+1}<x_{i j}^{(L)} \text { or } x_{i j}^{g+1}>x_{i j}^{(U)} \text {, then } \\
& \qquad x_{i j}^{g+1}=\operatorname{rand}[0,1] \cdot\left(x_{i j}^{(U)}-x_{i j}^{(L)}\right)+x_{i j}^{(L)} .
\end{aligned}
$$

3.2. Deformed Differential Evolution Algorithm. In the practical application, the differential evolution algorithm is developed for the convenience of representation, which is described in the form of $\mathrm{DE} / \mathrm{x} / \mathrm{y} / \mathrm{z}$.

The letter $\mathrm{x}$ represents the selection method of the base vector (the mutant target individual) of the mutation operation, and the letter $\mathrm{x}$ can be "rand" (representing a randomly selected individual from the population) or "best" (representing the best selected individual from the population). used.

The letter y represents the number of differential vectors

The letter $\mathrm{z}$ represents the crossover method and is usually performed by Bernoulli experiment method with "bin."

In accordance with the above provisions, the aforementioned differential evolution algorithm can be expressed as $\mathrm{DE} / \mathrm{rand} / 1 /$ bin. Storn and Price proposed DE extension mode as follows [7]:

Mode 1: DE/best/1/bin:

$$
v_{i}^{g}=x_{\text {best }}^{g}+F \cdot\left(x_{r_{1}}^{g}-x_{r_{2}}^{g}\right) .
$$

Mode 2: DE/rand-to-best/1/bin:

$$
v_{i}^{g}=x_{i}^{g}+\lambda \cdot\left(x_{\text {best }}^{g}-x_{i}^{g}\right)+F \cdot\left(x_{r_{1}}^{g}-x_{i}^{g}\right) .
$$

Mode 3: DE/best/2/bin:

$$
v_{i}^{g}=x_{\text {best }}^{g}+F \cdot\left(x_{r_{1}}^{g}-x_{r_{2}}^{g}+x_{r_{3}}^{g}-x_{r_{4}}^{g}\right) .
$$

Mode 4: DE/rand/2/bin:

$$
v_{i}^{g}=x_{r_{5}}^{g}+F \cdot\left(x_{r_{1}}^{g}-x_{r_{2}}^{g}+x_{r_{3}}^{g}-x_{r_{4}}^{g}\right) .
$$

Different deformation modes have their own characteristics, but through a large number of experimental studies,
Storn and Price show that the performance of DE/best/2/bin and $\mathrm{DE} / \mathrm{rand} / 1 / \mathrm{bin}$ is better than other methods, and $\mathrm{DE} /$ best/2/bin and $\mathrm{DE} / \mathrm{rand} / 1 /$ bin are the most widely applied DE extension modes in the actual engineering design process.

3.3. Improved Differential Evolution Algorithm. In view of the disadvantages such as slow convergence speed and the decrease of population diversity, the algorithm is improved by using the information and target information to improve the spatial characteristics and the complex optimization environment. The uniform distribution proposed by [17] can completely characterize the solution space and is conducive to the enhancement of population diversity, and the uniformity of differential evolution operator is studied. Through the analysis, individuals with uniform distribution can be obtained after mutation and selection operations, and those crossover operators that cannot exhibit properties of uniform distribution are improved to obtain the improved crossover operators with uniform distribution according to uniform distribution theory. Secondly, the transformation function is used to transform the original function, so that the current local minimal point and the region of the inferior to the point can be stretched to a certain height and keep the optimized function value below the current local minimal point unchanged. So the number of local minimal points can be eliminated, and then the difficulty of subsequent search can be effectively reduced. At last, a uniform differential evolution algorithm based on transformation function for fast solving complex optimization problem is obtained (abbreviated to AtDE).

In the $\mathrm{DE}$ algorithm, the variable to be optimized can be directly processed as the algorithm individual. Let $x_{i}^{g}(i=$ $1,2, \ldots, n$ ) be $n$ individuals of the $g$ th generation (each individual has a $D$ dimensional real valued information), and then these individuals constitute the population $X^{g}$ of the $g$ th generation. After the initialization of the algorithm, all the individuals in the solution space are consistent with the uniform probability distribution. Through a series of mutation (denoted by $M_{g}$ ), crossover (denoted by $C_{g}$ ), and selection (denoted by $S_{g}$ ) operations, population evolution is ultimately completed. Because the uniformly distributed population can fully characterize solution space features during search procedure, it is conducive to enhance the diversity of population. DE algorithm based on the greedy criterion compares the intermediate individual $u_{i}^{g}$ with the target individual $x_{i}^{g}$ in the current population to determine whether the intermediate individual $u_{i}^{g}$ can become a member of the next generation. In the process of running, the algorithm keeps searching for the extreme value information. If the maximum information $f\left(x^{b}\right)$ is not changed after two iterations (which can be set according to the complexity of the trial function), the algorithm is considered to be a local minimum, and according to this information the solution space is divided into $S_{1}=\left\{x \mid f(x) \geq f\left(x^{b}\right)\right\}$ and $S_{2}=$ $\left\{x \mid f(x)<f\left(x^{b}\right)\right\}$. Therefore, the transformation function is used to simplify the complex optimization environment and eliminate the search area worse than the local extreme point, while the search area better than the local extreme point 
remains unchanged. Hence the algorithm more easily gets rid of the local extreme points of the optimization problem, and it cooperates with the distribution of the individual's uniformity to guarantee the algorithm converge to the global optimal solution of the problem.

According to the first two sections, the specific steps of the AtDE algorithm are as follows.

Step 1. Set $g=0$, and create initial population.

Step 2. Calculate the fitness of each individual in the population.

Step 3. Evaluate whether the optimal individual is located in the local minimum. If yes, then let $f(x)=\varphi(x)$; else go to the next step.

Step 4. If the shutdown conditions are met, then shut the algorithm down; else go to the next step.

Step 5. Perform mutation operation $M_{g}$, and get the intermediate population $V_{g}$.

Step 6. Use uniform crossover operator for the original population and the intermediate population, and get the new intermediate population $U_{g}$.

Step 7. Get the new population of the next generation by operator selection.

Step 8. Set $g=g+1$, and then go to Step 2 .

\section{Rolling Force Prediction Model Based on Uniform Differential Neural Network}

4.1. Application Strategy. Because the mathematical model has a solid theoretical basis and it can roughly predict the changing trend of the rolling force, the prediction model of the combination of the mathematical model and the uniform differential neural network is used in the setting calculation. The prediction model is shown as follows:

$$
F=F_{0}+F_{\mathrm{NN}},
$$

where $F_{0}$ is the calculated value of rolling force and $F_{\mathrm{NN}}$ is the difference between the calculated value and the actual value of rolling force for uniform differential neural network.

The advantages of the algorithm are as follows: on the one hand, because of the introduction of a neural network model with strong nonlinear approximation ability, the relationship between the parameters and the rolling force is well described, moreover, by online learning and real-time feedback correction, the model further improves the adaptability of the rolling force online model to the parameters variation and random disturbance, and then the prediction accuracy of the model is improved; on the other hand, because of the existence of the conventional model, the adaptive learning speed is improved, and the increment of the control of the intermediate variable is guaranteed not to be too large, so the stability of the rolling force prediction is improved, and the

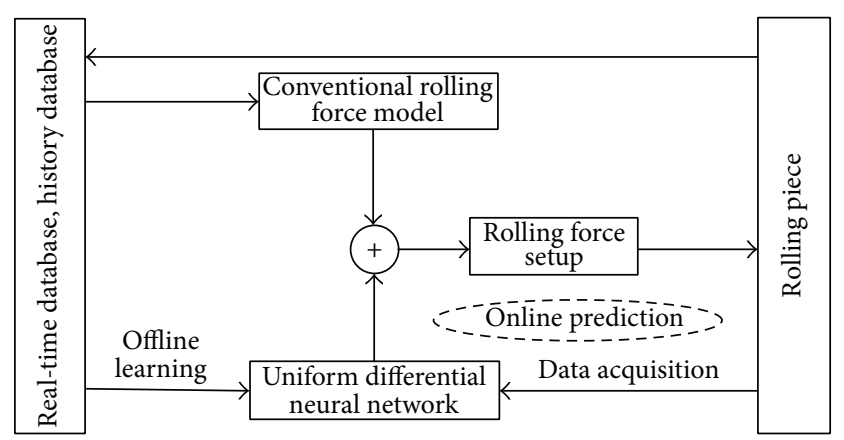

FIGURE 3: Application strategy of uniform differential neural network rolling force prediction model.

prediction accuracy of the rolling force is further improved. The application strategy of uniform differential neural network rolling force prediction model is shown in Figure 3.

\subsection{Structure Design}

4.2.1. Design of Input Layer and Output Layer. The input and output layer node number of neural network is designed according to the requirement of the user. On the basis of the information required to ensure the accuracy of network prediction model, the system size should be reduced as much as possible so as to reduce the learning time and the complexity of the system. The basic form of rolling force mathematical model can be expressed as $F=f\left(B, R^{\prime}, h_{\text {in }}, h_{\text {out }}, K_{m}, Q_{p}\right)$, which means the rolling force is associated with the strip width, entrance thickness, exit thickness, roll diameter, stress state, and deformation resistance coefficient, while, according to the spring equation, the exit thickness is associated with the setting value of the roll gap. The stress state coefficient $Q_{p}$ is related to flattening roll diameter and reduction rate. The deformation resistance $K_{m}$ is related not only to the chemical composition of the metal materials but also to the plastic deformation (deformation temperature, deformation velocity, and deformation degree). Combined with these factors, the rolling force can be expressed as follows:

$$
F=f\left(B, R, h_{\text {in }}, \varepsilon, S, C \%, \operatorname{Si} \%, \mathrm{Mn} \%, T, u_{m}\right),
$$

where $\mathrm{C} \%, \mathrm{Si} \%$, and $\mathrm{Mn} \%$ are the contents of $\mathrm{Mn}, \mathrm{Si}$, and $\mathrm{C}$ in raw materials, respectively, which can reflect the characteristics of the material.

Considering that the working roll radius is relatively stable in the process of steady state rolling, in order to reduce the complexity of the network, the parameter $R$ is not set as the network input.

Therefore, the following nine main factors affecting the rolling force are set as the input parameters of the network: the entrance thickness, the reduction rate, the roll gap, the entrance width, the rolling speed, the rolling temperature, and contents of $\mathrm{C}, \mathrm{Si}$, and $\mathrm{Mn}$, respectively. There is only one node in the output layer, which is the deviation between the calculated value and the actual value of the mathematical model. 
4.2.2. Precision of Network Training. The goal of improving the accuracy of the network can be achieved by increasing the number of hidden layers as well as the number of hidden layer nodes. The latter is simpler in implementation, so in this paper the hidden layer is fixed to one layer, and only the node number of the single hidden layer is automatically optimized. The node activation functions of both the hidden layer and output layer are Sigmoid functions.

4.2.3. Data Processing. The error data and the noise data will be removed before training. At the same time, the different variables represent different physical quantities, and their range may vary greatly, so all the data must be normalized to the same range of values. Because the activation function is the Sigmoid function, in order to avoid working in the flat area of the function, the data need to be transformed to 0.10.9. For this purpose, the following transformation is done on the data of the training sample set and the prediction sample set [17]. The new variable $x_{c}$ is

$$
x_{c}=\frac{0.9-0.1}{x_{\max }-x_{\min }} x+\left(0.9-\frac{0.9-0.1}{x_{\max }-x_{\min }} x_{\max }\right) .
$$

After the weights are obtained by training uniform differential neural network, the output value of the output layer is obtained by using the prediction sample, and the rolling force deviation value must be obtained by the transformation:

$$
x=x_{\max }+1.25\left(x_{c}-0.9\right)\left(x_{\max }-x_{\min }\right),
$$

where $x_{\max }$ and $x_{\min }$ are the maximum and minimum values of the normalized variables, respectively.

4.2.4. Parameter Setting. By using the uniform differential evolution algorithm, the code string for optimizing the structure and weights of the neural network consists of five parts: the number of the hidden nodes, the connection weights between the input layer and the hidden layer, the connection weights between the hidden layer and the output layer, the hidden layer threshold, and the output layer threshold. Set the numbers of input units, hidden units, and output units of the single hidden layer network to be $m, p$, and $q$, respectively.

Because the number of the hidden nodes is indefinite, the length of code string is variable during network optimization, and inconvenience will be brought to the operator's operation. At first, the maximum possible length of the code string is chosen to determine the maximum possible value $p_{\max }$ and then gradually reduced to determine the optimal hidden layer node. In this paper, $p_{\max }=2(m+q)$. According to the actual need, if we assume that the neurons of each layer are only connected to the neurons of its previous layer and there is no direct connection between the input and output, then the total number of connection weights is $(m+q) p_{\max }$.

The total length of code string $H$ is

$$
H=(m+q+1) p_{\max }+q+1 .
$$

The total length of the code string $H$ includes all information about network structure, connection weights, and threshold values. The structure of uniform differential neural network code strings is shown as follows:

$$
\begin{gathered}
\frac{H(1), H(2), \ldots, H(p)}{\text { Number of hidden nodes }} \\
\frac{W_{i j}(i=1,2, \ldots, m ; j=1,2, \ldots, p)}{\text { Weights of input layer and hidden layer }} \\
\frac{V_{j t}(j=1,2, \ldots, p ; t=1,2, \ldots, q)}{\text { Weights of hidden layer and output layer }} \\
\frac{\theta_{j}(j=1,2, \ldots, p)}{\text { Hidden layer threshold }} \\
\frac{\gamma_{t}(t=1,2, \ldots, q)}{\text { Output layer threshold }} .
\end{gathered}
$$

Let the sample pair of training set be the input and the expected output of the uniform differential neural network, calculate the error between the network output and the expected output, and take the error sum of squares as the fitness function $J[18]$ :

$$
J(n)=\sum_{k=1}^{N} \sum_{j=1}^{q}\left(y_{j}(k)-\widehat{y}_{j}(k)\right)^{2},
$$

where $n$ is the $n$th individual in the population, $N$ is the number of the sample sets of network input and output, $q$ is the number of output layer nodes, $y_{j}(k)$ is expected output of the $j$ th output node when the $k$ th sample is input, and $\widehat{y}_{j}(k)$ is actual output of the $j$ th output node when the $k$ th sample is input.

The other parameter settings of the uniform DE algorithm are as follows. The population size is 60 , the maximum evolution generation is $2000, \mathrm{CR}=0.8$, and $F=0.5$.

The schematics of uniform DE algorithm optimizing neural network are shown in Figure 4.

\section{Simulation}

After the data collection of a plate mill for a period of time, 300 sets of data with good shape are selected to form a set of training samples. The structural parameters and the weight thresholds of the neural network are optimized by the uniform DE algorithm. The number of the hidden layer neurons is 11, and Table 1 shows the weights of the input layer and the hidden layer of the neural network.

At the same time, the weights of the hidden layer and the output layer are $0.3193,-0.7575,-0.3996,-0.4072,-0.6635$, $0.0252,0.0334,0.8492,0.4070,-0.4100$, and -0.5028 , the hidden layer thresholds are $-0.0879,-0.3755,-0.0685,0.7466$, $-0.1059,0.1128,0.4648,0.4294,0.8185,-0.5616$, and 0.4474 , and the output layer threshold is 0.1540 .

The neural network optimized by uniform differential algorithm can effectively avoid falling into local minima and speed up the training speed of the network. As shown in Figure 5, in the same accuracy (0.0045) requirements, the AtDE neural network uses 1430 iterations, and BP neural 


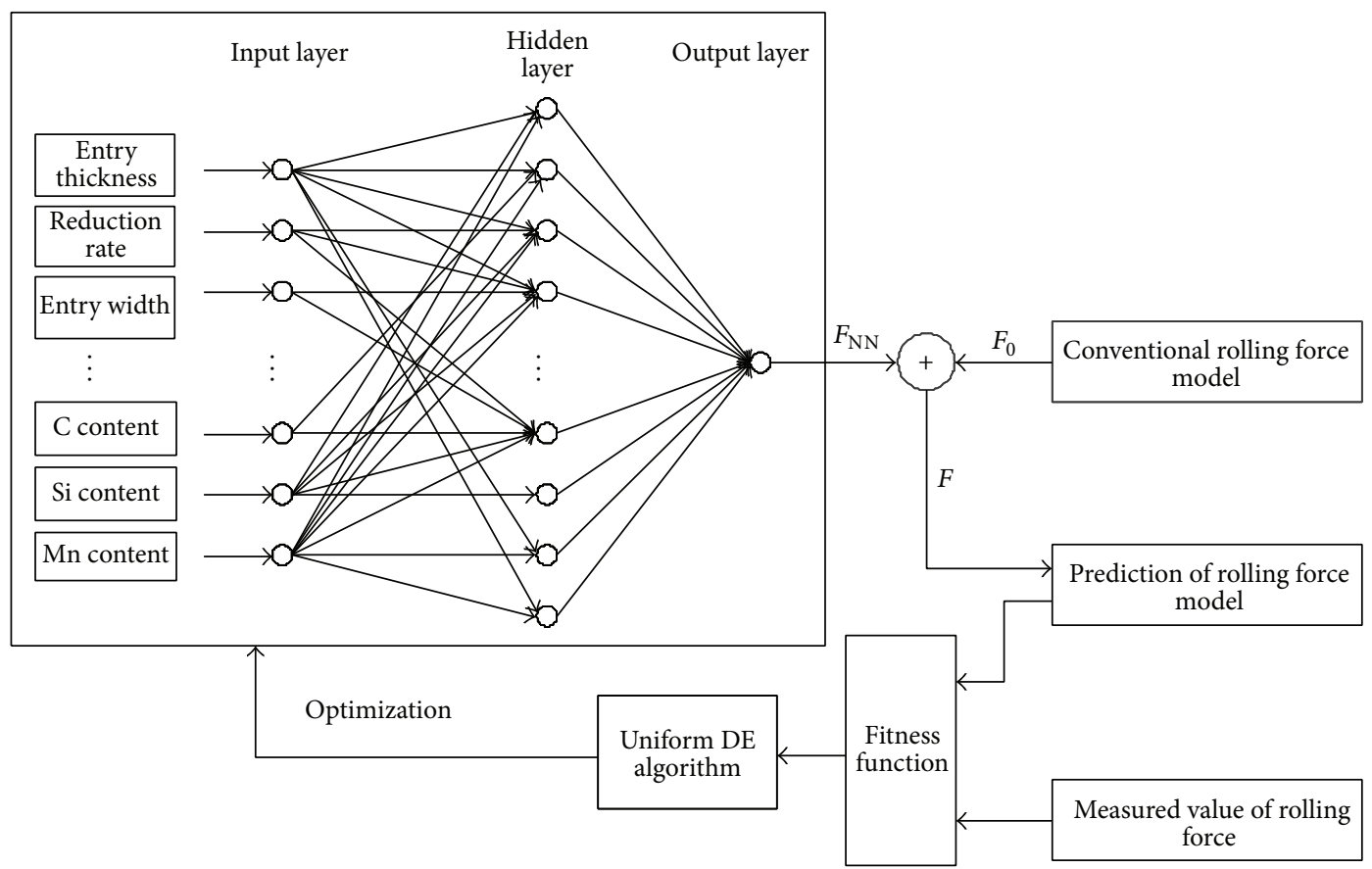

FIGURE 4: Schematics of uniform DE algorithm optimizing neural network.

TABLE 1: Optimized input-to-hidden layer weights.

\begin{tabular}{|c|c|c|c|c|c|c|c|c|c|}
\hline \multirow{2}{*}{$p$} & \multicolumn{9}{|c|}{$m$} \\
\hline & 1 & 2 & 3 & 4 & 5 & 6 & 7 & 8 & 9 \\
\hline 1 & 0.4456 & -0.2414 & 0.1351 & -0.0842 & -0.4818 & 0.1771 & 0.9440 & -0.0075 & -0.4579 \\
\hline 2 & 0.0448 & 0.1204 & -0.0670 & -0.7016 & 0.7175 & 0.1968 & -0.4644 & 0.7049 & 0.9254 \\
\hline 3 & -0.3353 & 0.1644 & -0.7372 & 0.2863 & 0.0220 & 0.5553 & -0.3442 & -0.1290 & 0.5077 \\
\hline 4 & -0.2948 & 0.1012 & -0.2111 & -0.5865 & 0.0200 & 0.4889 & 0.2332 & -0.5547 & 0.6458 \\
\hline 5 & 0.4114 & -0.4637 & 0.1488 & 0.2867 & 0.0850 & 0.0605 & 0.1048 & -0.8742 & 0.8624 \\
\hline 6 & 0.8308 & 0.8013 & -0.7589 & 0.2929 & 0.6459 & -0.6507 & 0.0033 & -0.0798 & -0.6997 \\
\hline 7 & 0.4553 & -0.5299 & -0.3334 & 0.6369 & -0.3279 & 0.1081 & 0.1722 & 0.7468 & 0.3356 \\
\hline 8 & 0.7016 & -0.1118 & 0.8840 & -0.1757 & -0.3861 & 0.2471 & -0.0591 & 0.4828 & -0.3004 \\
\hline 9 & 0.4182 & 0.2965 & -0.3703 & -0.3109 & -0.5969 & 0.0480 & 0.6277 & 0.3526 & -0.5174 \\
\hline 10 & 0.3091 & 0.6404 & -0.7189 & 0.2736 & -0.0714 & 0.1908 & 0.0999 & -0.1387 & 0.8122 \\
\hline 11 & 0.0782 & -0.1496 & 0.3921 & 0.2717 & -0.8132 & -0.0330 & -0.3873 & -0.9612 & 0.2904 \\
\hline
\end{tabular}

network uses at least 2000 iterations, which shows that the former is faster than the latter.

According to the network structure of the above, the 80 sets of data are selected as the trial sample. As shown in Figure 5, the accuracy of AtDE network is significantly higher than that of the conventional rolling force model, and the prediction error is within $5 \%$, which can meet the accuracy requirements of the rolling force prediction. Figure 6 shows that the rolling force prediction value of the AtDE neural network model is more close to the actual value than that of the conventional model.

\section{Conclusion}

In this paper, a uniform differential evolution algorithm with good performance in high dimensional function optimization is used to optimize the structure and weight value of neural network, so as to constitute a uniform differential neural network, and then it is used to improve the accuracy of rolling force prediction in the process control system of medium and heavy plate. Specific research contents are as follows:

(1) The basic theory and its algorithm improvement measures of BP neural network are analyzed. 


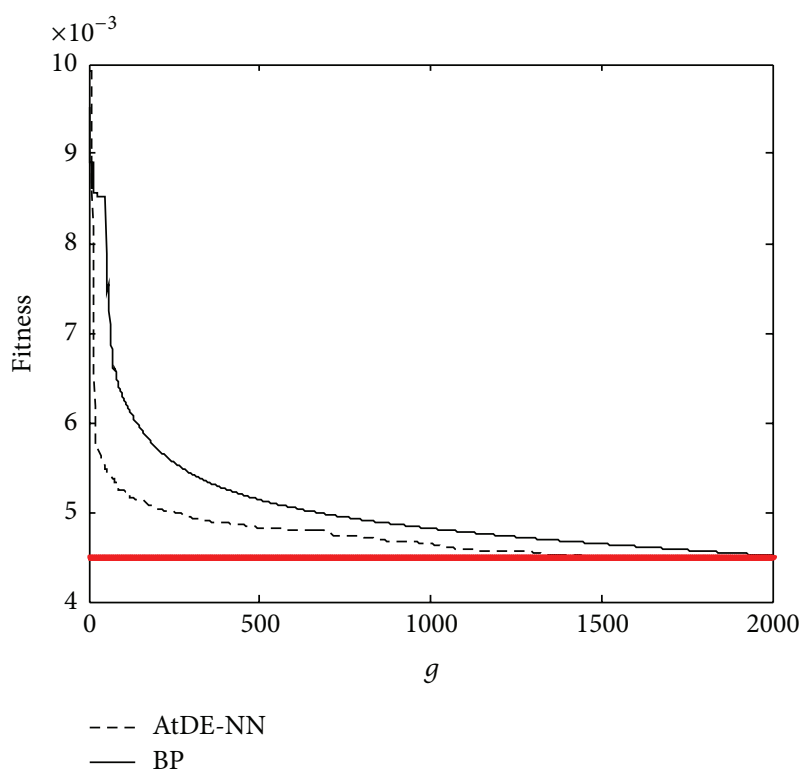

FIGURE 5: Convergence speed comparison between BP neural network and AtDE neural network.

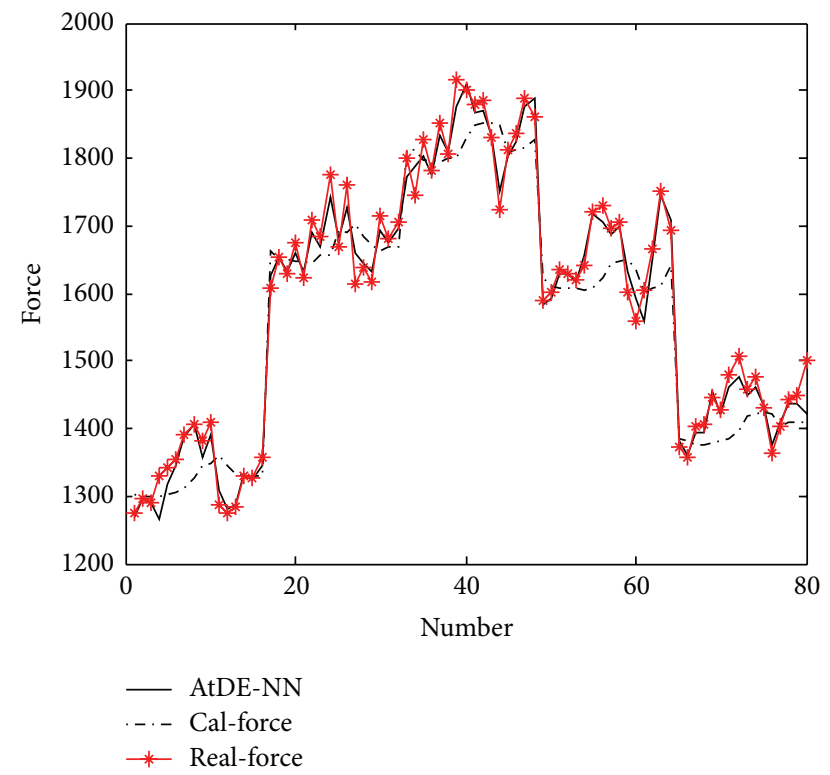

FIgURE 6: Comparison between AtDE neural network model and the conventional model of rolling force prediction value and the measured value.

(2) In view of some defects of BP neural network, the AtDE algorithm is constructed, and the structure parameters and the weights of the neural network are optimized by using uniform DE algorithm, accordingly the performance of the neural network is improved, and the algorithm foundation of the adaptive learning of plate rolling force is laid.

(3) On the basis of analysis of the traditional rolling force prediction model, the method of applying neural network to the rolling force prediction is discussed, and the rolling force prediction model based on uniform differential neural network is established. The results show that the model can improve the prediction accuracy.

\section{Competing Interests}

The authors declare that there is no conflict of interests regarding the publication of this paper.

\section{Acknowledgments}

This work is partially supported by National Natural Science Funds of China (51404021), Beijing Municipal Natural Science Foundation (3154035), and the Fundamental Research Funds for the Central Universities (FRF-TP-15-061A3).

\section{References}

[1] D. M. Lee and S. G. Choi, "Application of on-line adaptable Neural Network for the rolling force set-up of a plate mill," Engineering Applications of Artificial Intelligence, vol. 17, no. 5, pp. 557-565, 2004.

[2] H. N. Bu, Z. W. Yan, C. M. Zhang, and D. H. Zhang, "Comprehensive parameters adaption of rolling force model based on objective function in tandem cold mill," Applied Mechanics and Materials, vol. 551, pp. 296-301, 2014.

[3] Z. Zhao, J. Yang, H. Che, H. Sun, and H. Yang, "Application of artificial bee colony algorithm to select architecture of a optimal neural network for the prediction of rolling force in hot strip rolling process," Journal of Chemical and Pharmaceutical Research, vol. 5, no. 9, pp. 563-570, 2013.

[4] R. Heeg, A. Kugi, O. Fichet, L. Irastorza, and C. Pelletier, "Modeling and control of plate thickness in hot rolling mills," Proceedings of the 16th IFAC World Congress, vol. 16, part 1, pp. 1680-1685, 2005.

[5] "Sumitomo metal plate production technology and product development," Sumitomo metal, no.1, 1998.

[6] A. Wacher and B. R. Seymour, "A radiation model of a rapid thermal processing system," Mathematics-in-Industry Case Studies Journal, vol. 3, pp. 1-18, 2011.

[7] R. Storn and K. Price, "Differential evolution-a simple and efficient adaptive scheme for global optimization over continuous spaces," Tech. Rep. TR-95-012, International Computer Science Institute, Berkeley, Calif, USA, 1995.

[8] S. Das and P. N. Suganthan, "Differential evolution: a survey of the state-of-the-art," IEEE Transactions on Evolutionary Computation, vol. 15, no. 1, pp. 4-31, 2011.

[9] F. Neri and V. Tirronen, "Recent advances in differential evolution: a survey and experimental analysis," Artificial Intelligence Review, vol. 33, no. 1-2, pp. 61-106, 2010.

[10] Y.-J. Zheng, X.-L. Xu, H.-F. Ling, and S.-Y. Chen, "A hybrid fireworks optimization method with differential evolution operators," Neurocomputing, vol. 148, pp. 75-82, 2015.

[11] M. Ali, P. Siarry, and M. Pant, "An efficient Differential Evolution based algorithm for solving multi-objective optimization problems," European Journal of Operational Research, vol. 217, no. 2, pp. 404-416, 2012.

[12] T. R. Bhat, D. Venkataramani, V. Ravi, and C. V. S. Murty, "An improved differential evolution method for efficient parameter 
estimation in biofilter modeling," Biochemical Engineering Journal, vol. 28, no. 2, pp. 167-176, 2006.

[13] Y. Cai, J. Wang, Y. Chen, T. Wang, H. Tian, and W. Luo, "Adaptive direction information in differential evolution for numerical optimization," Soft Computing, vol. 20, no. 2, pp. 465494, 2016.

[14] K. V. Price, "Differential evolution: a fast and simple numerical optimizer," in Proceedings of the Biennial Conference of the North American Fuzzy Information Processing Society (NAFIPS '96), pp. 524-527, New York, NY, USA, June 1996.

[15] R. Storn and K. Price, "Differential evolution-a simple and efficient heuristic for global optimization over continuous spaces," Journal of Global Optimization, vol. 11, no. 4, pp. 341359, 1997.

[16] P. H. Natvarlal, Differential Evolution A Method of Global Optimization, University of Texas, Arlington, Va, USA, 2002.

[17] X. F. Cai and S. N. Zhong, "Evolutionary uniform optimization algorithm," Journal of Mathematics, vol. 25, no. 3, pp. 349-354, 2005.

[18] T. Fechner, D. Neumerkel, and I. Keller, "Adaptive neural network filter for steel rolling," in Proceedings of the IEEE International Conference on Neural Networks and IEEE World Congress on Computational Intelligence, vol. 6, pp. 3915-3920, Orlando, Fla, USA, June-July 1994. 


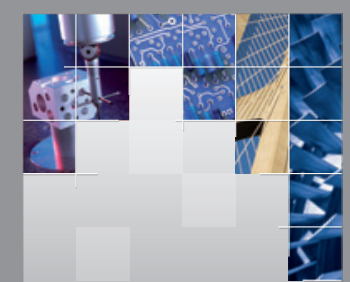

\section{Enfincering}
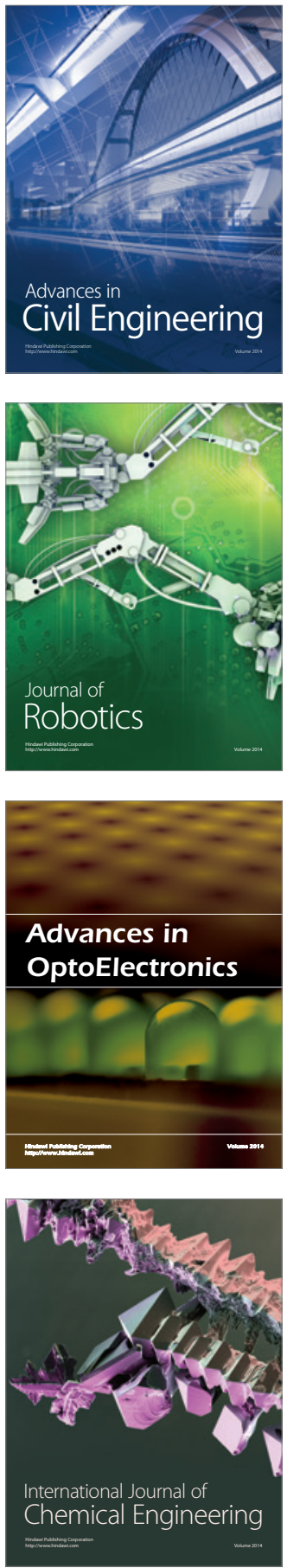

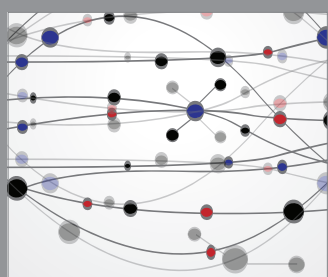

The Scientific World Journal

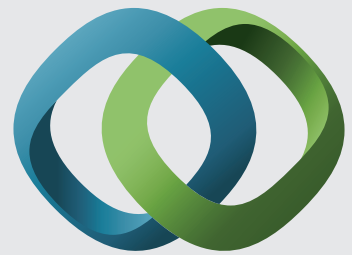

\section{Hindawi}

Submit your manuscripts at

http://www.hindawi.com
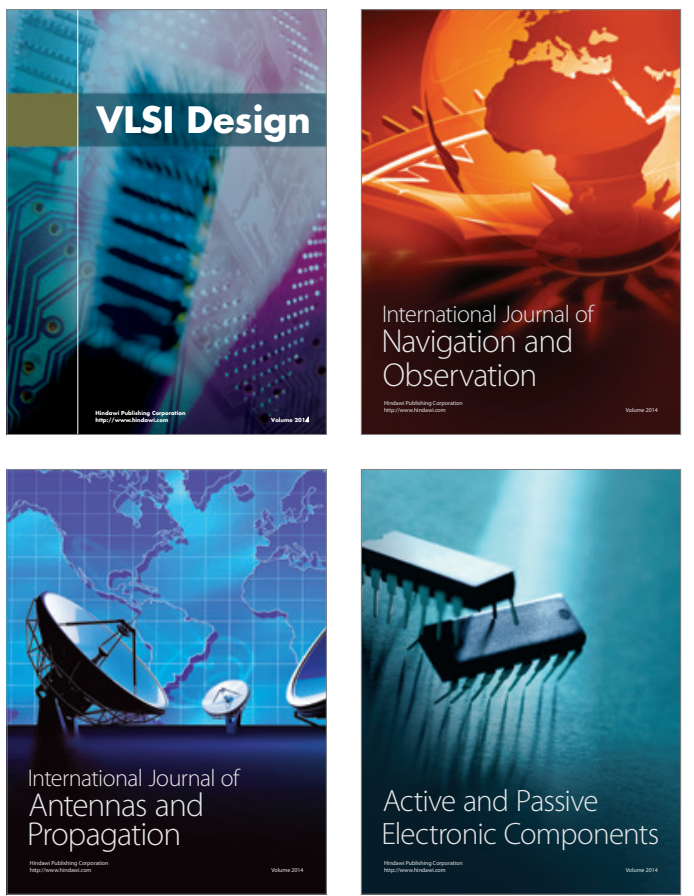
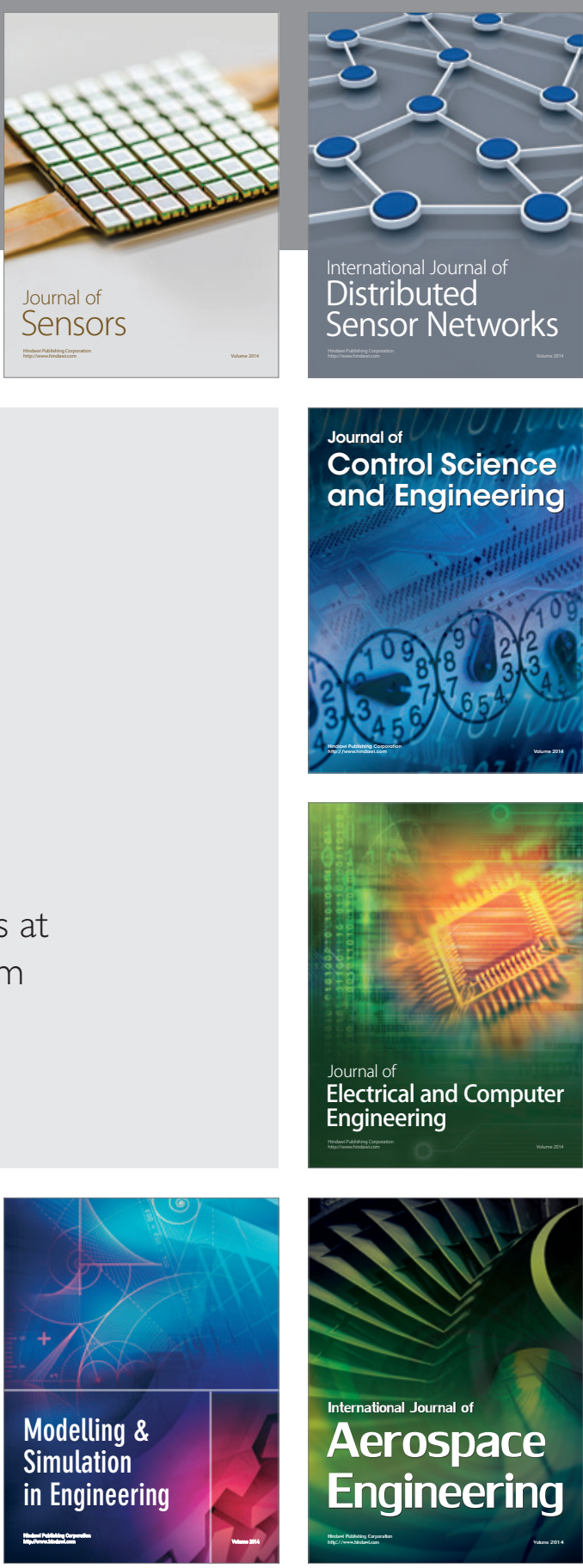

International Journal of

Distributed

Sensor Networks

Journal of

Control Science

and Engineering
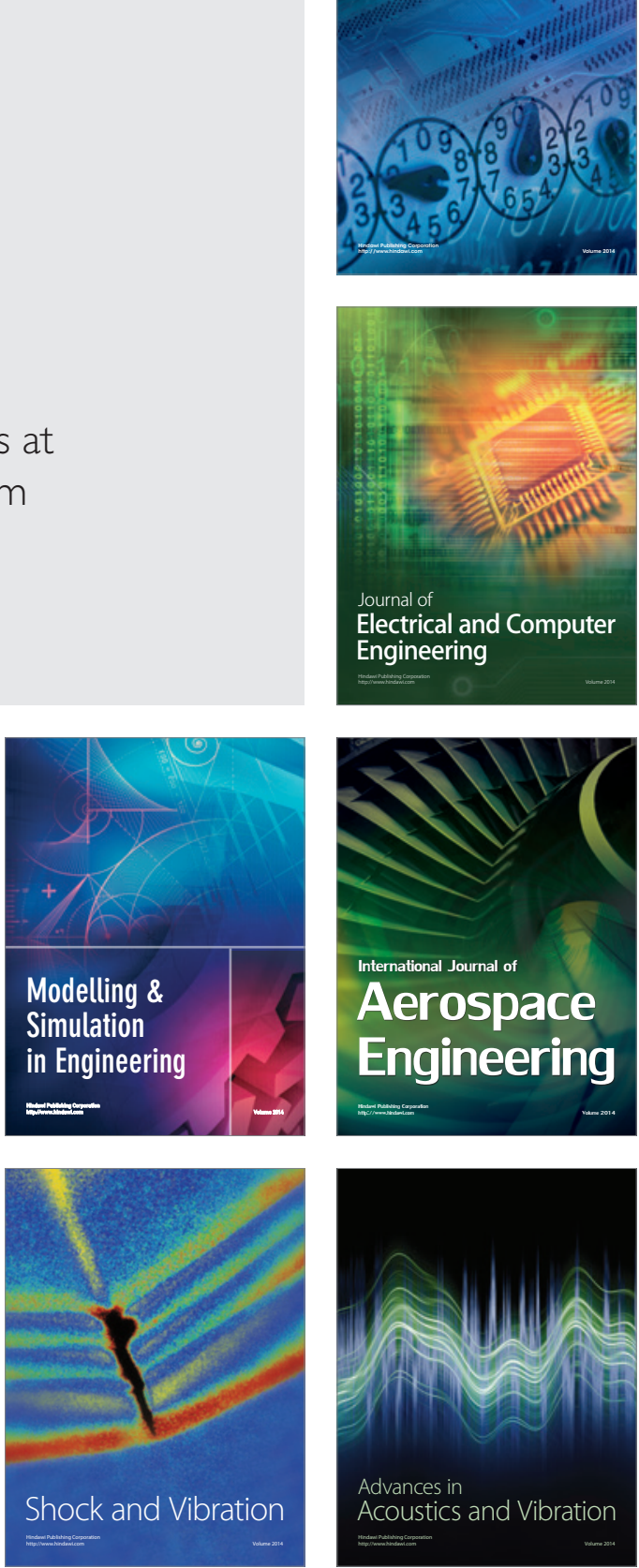